\title{
Clinically significant depressive symptoms and sexual behaviour among men who have sex with men
}

Ada R. Miltz, Alison J. Rodger, Janey Sewell, Andrew Speakman, Andrew N. Phillips, Lorraine Sherr, Richard J. Gilson, David Asboe, Nneka C. Nwokolo, Amanda Clarke, Mark M. Gompels, Sris Allan, Simon Collins and Fiona C. Lampe; for the AURAH Study Group

\section{Background}

The relationship between depression and sexual behaviour among men who have sex with men (MSM) is poorly understood.

\section{Aims \\ To investigate prevalence and correlates of depressive symptoms (Patient Health Questionnaire-9 score $\geq 10$ ) and the relationship between depressive symptoms and sexual behaviour among MSM reporting recent sex.}

\section{Method}

The Attitudes to and Understanding of Risk of Acquisition of HIV (AURAH) is a cross-sectional study of UK genitourinary medicine clinic attendees without diagnosed HIV (2013-2014).

\section{Results}

Among 1340 MSM, depressive symptoms (12.4\%) were strongly associated with socioeconomic disadvantage and lower supportive network. Adjusted for key sociodemographic factors, depressive symptoms were associated with measures of condomless sex partners in the past 3 months ( $\geq 2$ (prevalence ratio (PR) 1.42, 95\% Cl 1.17-1.74; $P=0.001$ ) unknown or HIV-positive status (PR 1.43, 95\% Cl 1.20-1.71; $P<0.001)$ ), sexually transmitted infection (STI) diagnosis
(PR 1.46, 95\% Cl 1.19-1.79; $P<0.001$ ) and post-exposure prophylaxis use in the past year (PR 1.83, 95\% Cl 1.33-2.50; $P<0.001)$.

\section{Conclusions}

Management of mental health may play a role in HIV and STI prevention.

\section{Declaration of interest}

A.N.P. has received payments for presentations made at meetings sponsored by Gilead in spring 2015. N.C.N. has received support for attendance at conferences, speaker fees and payments for attendance at advisory boards from Gilead Sciences, Viiv Healthcare, Janssen Pharmaceuticals and Bristol-Myers Squibb and a research grant from Gilead Sciences. D.A. served on the advisory board for Gilead in January 2016. M.M.G. has had sponsorship to attend conferences by Bristol-Myers Squibb, been on the Biocryst advisory board and run trials for Merck, Gilead, SSAT, Biocryst and Novartis.

\section{Copyright and usage}

(C) The Royal College of Psychiatrists 2017. This is an open access article distributed under the terms of the Creative Commons Non-Commercial, No Derivatives (CC BY-NC-ND) license.
HIV transmission is ongoing among gay, bisexual and other men who have sex with men (MSM) in the UK, with 3320 new diagnoses in 2015. ${ }^{1}$ Although HIV prevention has been a public health priority among MSM in the UK and Europe, mental health has not generally been a focus of European studies of HIV and sexually transmitted infection (STI) risk and prevention among MSM. The potential role of depression in sexual health and transmission of HIV is not well understood.

\section{Quantitative link in epidemiological literature}

Assessment of the association between depression and condomless sex (CLS) in epidemiological studies may be problematic, because individuals with depressive symptoms may be more likely to engage in CLS or, on the other hand, more likely to have lowered libido and less interest in sex. The direction in which the association operates may be a function of a number of factors including the severity of the symptoms or the type of coping mechanism employed by the individual. ${ }^{2}$ Such opposing trends could result in an observed overall lack of association. The only meta-analysis that has examined this association among MSM included studies conducted up to $1999 .{ }^{2}$ Findings across studies were inconsistent. In combined estimates, there was no significant relationship between depression and measures of CLS. Stratifying by sample population (MSM, substance users or other) and HIV status did not significantly moderate the association. A number of studies of MSM and HIV-negative MSM published since 1999 have reported a significant relationship between depressive symptoms and CLS in unadjusted analysis ${ }^{3-6}$ and after adjusting for sociodemographic, lifestyle, psychosocial and/or sexual behaviour measures. ${ }^{7-14}$ However, some studies have failed to find a relationship in unadjusted analysis ${ }^{15-20}$ or after adjusting for sociodemographic, lifestyle and psychosocial measures. ${ }^{21}$ The association and magnitude of effect may vary according to the setting and specific sample of MSM under study. The vast majority of studies have been conducted in the USA. To date, only two relatively small European studies have investigated the relationship between depression and CLS among MSM, both finding that depressive symptoms were associated with CLS measures. ${ }^{3,5}$

\section{Theoretical link in self-regulation and coping literature}

It has been suggested that some individuals may engage in CLS, not because they lack information or intentions to be safe, but because sexuality is a non-rational emotionally charged area. A number of factors may have an impact on non-rationality of sexual risk behaviour, such as psychological symptoms (e.g. depression may distort perceptions of personal vulnerability) and recreational drug use (which may lead to increased sexual arousal and disinhibition). However, it is also possible that any such associations are bidirectional; experiences of sexual risk may increase vulnerability to symptoms of depression and drug use. ${ }^{22}$

Cognitive escape is a theoretical process whereby individuals turn their attention away from threatening cues, completely or 
partially escaping from personal self-awareness. There are two potential pathways through which cognitive escape might lead to CLS. The first is fatalistic beliefs about HIV, the notion that acquiring HIV is inevitable. The second is 'determination' to perceive oneself to be at low risk, despite evidence to the contrary. ${ }^{23}$ Individuals with depression have an increased propensity to engage in escapism, characterised by fatalistic beliefs, as a coping strategy. ${ }^{7,22}$ The association between depression and CLS may also be due to the tendency of individuals with depressive symptoms to experience low self-efficacy. The principle of self-efficacy, as conceptualised in social cognitive theory, is that regardless of one's attitude, action (e.g. ensuring condom use during sex) requires additional confidence in one's ability to produce a certain outcome (e.g. sexual safety). ${ }^{24}$ Conceptually, cognitive escape and self-efficacy are linked. Low self-efficacy is thought to make escape tendencies more likely. It is possible that depression may lower one's perceived ability to exercise self-protective control in sexual situations where this is desired (lowered self-efficacy for sexual safety) and lead to cognitive escape. ${ }^{7}$ Recreational drug use may also play a role in these relationships. In addition to any direct effect on autonomic or central nervous system mechanisms, substances may be used strategically to induce a state of cognitive release in sexual settings. Men with depressive symptoms may be more likely to engage in recreational drug use, perhaps as a form of self-medication. ${ }^{22}$

\section{Study objectives}

This study uses data from the Attitudes to and Understanding of Risk of Acquisition of HIV (AURAH) Study of individuals attending genitourinary medicine (GUM) clinics across the UK. It investigates, among HIV-negative or unknown status MSM who reported recent sex: (1) the prevalence of depressive symptoms and the association with sociodemographic and lifestyle factors; (2) the relationship of depressive symptoms with CLS and other sexual behaviour measures, and the potential confounding effect of recreational drug use; (3) the association between depressive symptoms and measures of self-efficacy for sexual safety; and (4) the prevalence of treatment for depression, and association of treated depression with sexual behaviour.

Men who did not report any sex in the past 3 months were excluded from this analysis, because depression may also be linked to a lack of sexual activity, ${ }^{2}$ and it was of particular interest to compare those having condom-protected sex with those having CLS.

\section{Method}

AURAH Study is a cross-sectional, self-administered questionnaire study in individuals attending 20 GUM clinics across the UK from June 2013 to November $2014 .{ }^{25}$ Individuals aged 18 years or over without diagnosed HIV were eligible for inclusion. A total of 4380 eligible participants were approached over the study period, of whom 2630 completed the questionnaire (response rate: 60\%). The confidential, self-administered questionnaire included questions on sociodemographics, health, lifestyle and sexual behaviour.

Men were classified as MSM if they met at least one of the following criteria: (1) reported being gay or bisexual (including other plurisexual identity labels: pansexual, omnisexual, open or queer, i.e. identities that are not explicitly based on attractions to one sex or gender ${ }^{26}$ ), (2) reported anal sex with a man in the past 3 months or (3) reported having disclosed to their family, friends or workmates as being gay, bisexual and/or attracted to men.

\section{Clinically significant depressive symptoms}

The Patient Health Questionnaire-9 (PHQ-9) was used to measure the prevalence of depressive symptoms. ${ }^{27}$ A score of $\geq 10$ was considered to indicate clinically significant depressive symptoms; details given in Box 1. The score was further classified in terms of severity of depressive symptoms: moderate (10-14), moderately severe (15-19) and severe (20-27). In addition, participants were asked whether they were receiving medical treatment or therapy for depression.

\section{Sexual behaviour measures}

The questionnaire included questions about anal sex with men, and vaginal or anal sex with women in the past 3 months, and sex without a condom. Four measures of CLS (anal or vaginal) were defined: (1) CLS with one or more partners, (2) CLS with two or more partners, (3) CLS with unknown and/or HIV-positive status partner(s) (men who reported no CLS partners of unknown HIV status, and only one HIV positive CLS partner who was a long-term partner and with whom they 'thought the risks of catching HIV were low because their partner was taking ART' were not counted) and (4) receptive CLS with unknown HIV-status partner(s). All reports of CLS were included regardless of the partner's gender. Participants were asked whether they had been diagnosed with an STI in the past year. STI diagnosis was defined as report of one of four bacterial infections: gonorrhoea, chlamydia, syphilis or lymphogranuloma venereum (LGV). Furthermore, participants were asked whether they had taken antiretroviral drugs after CLS to reduce the risk of becoming infected with HIV, post-exposure prophylaxis (PEP), in the past year. The final two measures considered concerned partner numbers: reporting 11 or more new sexual partners in the past year and reporting group sex (sex with more than one other person on the same occasion) in the past 3 months.

\section{Measures of self-efficacy for sexual safety}

The questionnaire included a number of statements on attitudes towards condom use, with response options: strongly agree, tend to agree, undecided or no opinion or not relevant to me, tend to disagree, strongly disagree. The following statement was used to measure self-efficacy: 'I feel confident that, if I want to, I can make sure a condom is used during sex with any partner, in any situation'. Strong agreement with this statement was considered to indicate high self-efficacy for sexual safety. Agreement ('strong' or 'tend to') with the following statement was considered to indicate difficulty in negotiating condom use: 'I find it difficult to discuss condom use with any new sexual partner'.

Men were also asked reasons for non-condom use in the past 3 months, for which one or more of the following options could be

\section{Box 1 The Patient Health Questionnaire 9-item scale (PHQ-9) ${ }^{27}$}

for depressive symptoms

'Over the past 2 weeks, how often have you been bothered by any of the following problems?'

1 Little interest or pleasure in doing things.

2 Feeling down, depressed or hopeless.

3 Trouble falling or staying asleep, or sleeping too much.

4 Feeling tired or having little energy.

5 Poor appetite or overeating.

6 Feeling bad about yourself - or that you are a failure or have let yourself or your family down.

7 Trouble concentrating on things, such as reading the newspaper or watching television

8 Moving or speaking so slowly that other people could have noticed/ being so restless that it is hard to sit still.

9 Thoughts that you would be better off dead or of hurting yourself in some way.

Response options:

Not at all (coded as 0 )

Several days (coded as 1)

More than half the days (coded as 2)

Nearly every day (coded as 3) 
chosen: 'Didn't think about using a condom or did not have a condom', 'Don't like using condoms or it's more enjoyable/close without a condom', 'My partner didn't want to use a condom', 'Felt unable to discuss condom use', 'Got carried away or was under the influence of alcohol or drugs' and 'Difficult for me/partner to keep erection or ejaculate when using a condom'.

\section{Statistical analysis}

Associations were assessed by $\chi^{2}$ tests, $\chi^{2}$ tests for trend and Fisher's exact test (when expected numbers were small) for univariable analysis, and modified Poisson regression with a robust variance estimator to produce adjusted prevalence ratios (PRs) for multivariable analysis. ${ }^{28}$ PRs were chosen for their ease of interpretation. It should be noted that, where the prevalence of the different outcome measures differ, it is not appropriate to compare the magnitude of PRs for depression across these different outcome measures.

For assessment of the relationship of sociodemographic and lifestyle factors with depressive symptoms, associations are presented unadjusted, and adjusted for the following socio-demographic factors: age group $(<25,25-29,30-39$ or $\geq 40$ years), country of birth (UK born or non-UK born/missing), self-reported sexual identity (gay or bisexual/straight), university education (yes or no/missing), ongoing relationship (yes or no/missing) and study region (London, South or other - see Table 1 footnote for definition).

For assessment of the relationship of depressive symptoms with sexual behaviour measures, associations are presented unadjusted, adjusted for the above sociodemographic factors and finally adjusted for the above sociodemographic factors plus current smoking (yes or no/missing), higher risk alcohol consumption (yes or no/missing; see Table 1 for definition) and number of recreational drugs used in the past 3 months (none, 1 , $2-4$ or $\geq 5$ ), to assess the extent to which the associations were attenuated when accounting for smoking, drug and alcohol use.

Measures of self-efficacy for sexual safety were hypothesised a priori to be on the casual pathway between depressive symptoms and sexual risk behaviour. The association of depressive symptoms with self-efficacy measures and the association of self-efficacy measures with sexual behaviour measures were investigated.

Finally, the prevalence of treatment for depression was assessed, and the associations with sexual behaviour measures were assessed according to the presence or absence of depressive symptoms and treatment for depression.

The proportion of missing values was $<5 \%$ for all variables used in analyses; missing values were incorporated into specific categories for some variables (see Table 1). All analyses were performed by STATA statistical software ${ }^{29}$ and reported according to the STROBE guidelines. ${ }^{30}$ Several sensitivity analyses were undertaken: (1) adjusting for age grouped into six categories $(<25,25-29,30-34$, $35-39,40-44$ or $\geq 45$ years), (2) adjusting for age as a continuous variable, (3) excluding missing values when defining each variable and (4) assessing the association between depressive symptoms and sexual behaviour measures only among men who reported anal sex with a man in the past 3 months. In each case, the findings were very similar to the main analysis (results not shown).

\section{Ethical approval}

The research protocol and all versions of the study documents were approved by the designated research ethics committee (NRES committee London-Hampstead, ref: 13/LO/0246).

\section{Results}

In total, 2630 individuals participated in the AURAH Study, of whom 1484 were defined as MSM. The current analysis is based on 1340 MSM who reported anal or vaginal sex in the past 3 months. Of these, 1238 (92.4\%) reported anal sex with men only, $66(4.9 \%)$ reported sex with both men and women and $36(2.7 \%)$ reported sex with women only in the past 3 months. Eighty-nine per cent of the 1340 men identified as gay, $9.5 \%$ as bisexual or another plurisexual identity label and $1.4 \%$ as straight. Eighty-two per cent of men were of White ethnicity; median age was 31 years. Overall, $57 \%$ were born in the UK, $67 \%$ had a university degree and $76 \%$ were attending a clinic in London (Table 1).

\section{Depressive symptoms}

The prevalence of depressive symptoms (PHQ-9 score $\geq 10$ ) was $12.4 \%$ (166 of 1340). Of these 166 men, 55\% had moderate depressive symptom severity, $30.1 \%$ had moderately severe symptoms and $15.1 \%$ had severe symptoms of depression.

\section{Correlates of depressive symptoms}

In unadjusted analysis, depressive symptoms were strongly associated with indicators of lower socioeconomic status (financial hardship, non-employment, rented or unstable housing and non-university education), and there was an exceptionally strong association with lower reported levels of a supportive network. Depressive symptoms were also associated with younger age, bisexual (or other plurisexual) identity, Midlands or Yorkshire or Humber region of recruitment, disclosing being MSM to few or no work colleagues, smoking, higherrisk drinking and greater number of recreational drugs used (Table 1). After adjusting for key sociodemographic factors, these associations remained, with some attenuation in the magnitude of effect, with the exception of study region and smoking, which were no longer significantly associated with depressive symptoms (Table 1).

\section{Sexual behaviour measures}

Overall, 63.7\% ( $n=853)$ of men reported CLS in the past 3 months. Of these, $91.7 \%(n=782)$ had CLS with men only, 3.9\% $(n=33)$ had CLS with both men and women and $4.5 \%(n=38)$ had CLS with women only. The prevalence of CLS and other sexual behaviour measures is presented in Table 2 . The prevalence of high selfefficacy was $67.2 \%(n=900)$ and $10.6 \%(n=142)$ for difficulty negotiating condom use. Among the 853 men who reported CLS, the reasons indicated for non-condom use (788 total responses) were as follows: got carried away or under the influence of alcohol or drugs $(n=369 ; 43.3 \%)$, don't like or more enjoyable without ( $n=353 ; 41.4 \%)$, their or their partner's difficulty in keeping an erection or ejaculating $(n=205 ; 24.0 \%)$, partner not wanting to $(n=147 ; 17.2 \%)$, didn't think about it or didn't have $(n=131$; $15.4 \%)$, and felt unable to discuss ( $n=37 ; 4.3 \%)$.

\section{Relationship between depressive symptoms and sexual behaviour}

Men with depressive symptoms $(n=166)$ were more likely than those without $(n=1174)$ to report measures of CLS partners in the past 3 months: $\geq 1$ (74.7\% v. $62.1 \%), \geq 2$ (44.6\% v. 30.4\%), unknown and/or HIV-positive status (50.6\% v. 33.2\%), receptive unknown status $(22.9 \%$ v. $12.7 \%)$. They were also more likely to report diagnosed bacterial STI $(42.2 \%$ v. $30.1 \%)$ and PEP use $(23.5 \% v$. $14.3 \%)$ in the past year $\left(P<0.01\right.$ for all, $\chi^{2}$ tests). The prevalence of CLS with one or more partners was $70.3 \%$ among men with moderate depressive symptom severity, $78.0 \%$ among men with moderately severe symptoms and $84.0 \%$ among men with severe symptoms of depression.

After adjusting for key sociodemographic factors, depressive symptoms were associated with all measures of CLS partners $(\geq 1$ (PR 1.18, 95\% CI 1.06-1.30; $P=0.002$ ), $\geq 2$ (PR 1.42, 95\% CI 1.171.74; $P=0.001$ ), unknown and/or HIV-positive status (PR 1.43, 95\% CI $1.20-1.71 ; P<0.001$ ), receptive unknown status (PR 1.60, 95\% CI 1.14-2.24; $P=0.006)$ ) and with bacterial STI diagnosis 


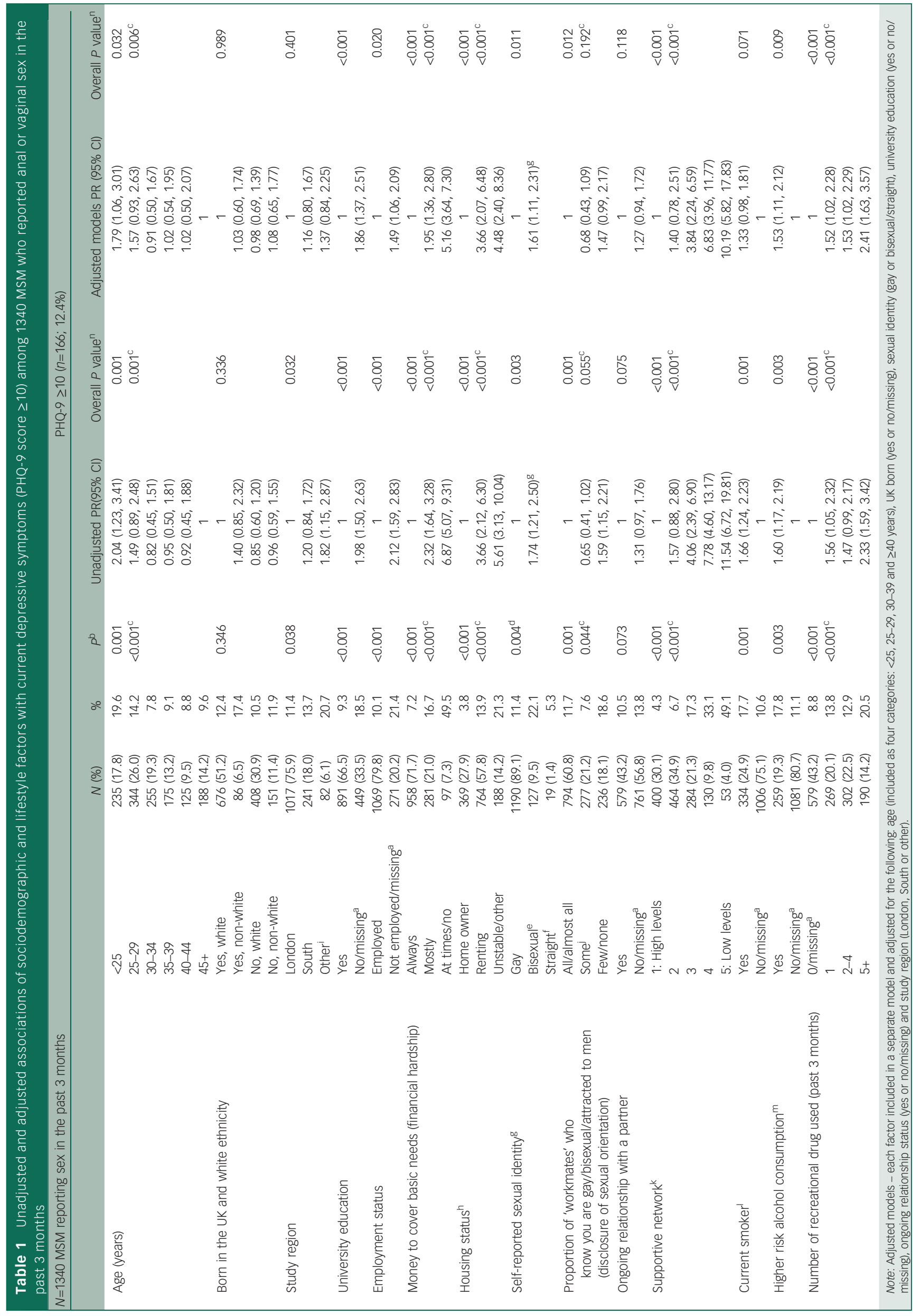




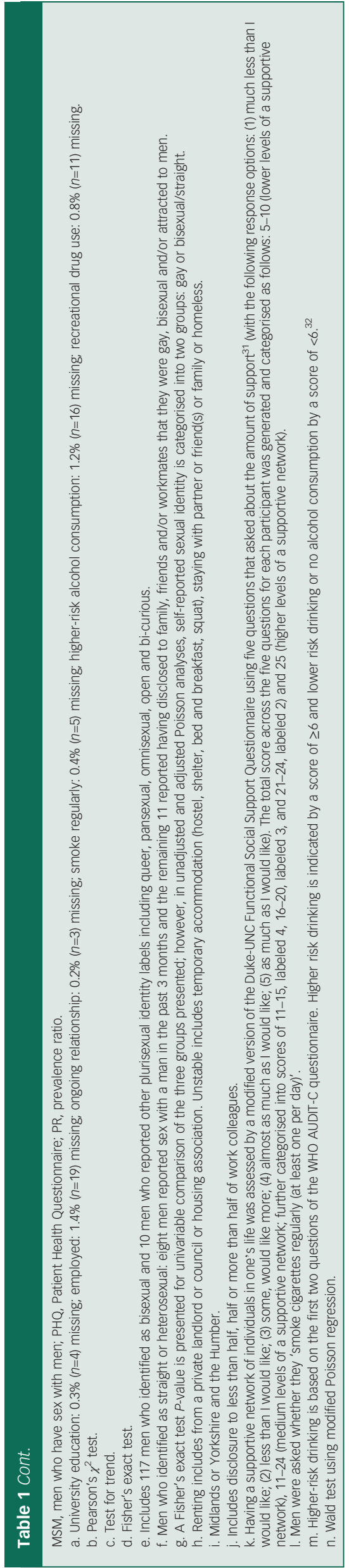

(PR 1.46, 95\% CI 1.19-1.79; $P<0.001$ ) and PEP use (PR 1.83, 95\% CI 1.33-2.50; $P<0.001$ ) (Fig. 1). After adjusting additionally for smoking, alcohol and recreational drug use, most of these associations were attenuated to some extent, but they remained significant (Fig. 1). Associations were also apparent among men who had not taken recreational drugs in the past 3 months (data not shown).

Depressive symptoms were not associated with having 11 or more new sexual partners in the past year or with group sex in the past 3 months, in unadjusted or adjusted analyses (Fig. 1).

\section{Self-efficacy for sexual safety: relationship with depressive symptoms and sexual behaviour}

Men with depressive symptoms were less likely to report high selfefficacy for sexual safety $(56.6 \% v .68 .7 \% ; P=0.002)$ and more likely to report difficulty negotiating condom use $(18.7 \%$ v. $9.5 \%$; $P<0.001)$ than men without. Adjusted for key sociodemographic factors, depressive symptoms were inversely associated with selfefficacy (PR 0.82, 95\% CI 0.71-0.94; $P=0.006$ ) and were associated with difficulty negotiating condom use (PR 1.77, 95\% CI 1.18-2.63; $P=0.005$ ). These associations remained with some attenuation, after additional adjustment for smoking, alcohol and recreational drug use (data not shown).

Men with high self-efficacy were less likely than those without to report CLS partners: $\geq 1$ ( $55.7 \%$ v. $80.0 \%), \geq 2$ ( $24.3 \%$ v. $48.0 \%)$, unknown and/or HIV-positive status (26.9\% v. 52.7\%), receptive unknown status $(10.3 \%$ v. $21.4 \%)$ and STI diagnosis $(29.2 \% v$. $36.4 \%$ ), and PEP use (13.6\% v. 19.3\%); $P \leq 0.01$ for all. Men who reported difficulty negotiating condom use were more likely than those who did not to report CLS partners: $\geq 1$ (80.3\% v. 61.7\%), $\geq 2$ (45.8\% v. 30.5\%), unknown and/or HIV-positive status (55.6\% v. $33.0 \%)$ and receptive unknown status (23.2\% v. $12.9 \%) ; P \leq 0.001$ for all. Adjusted for key sociodemographics (and additionally adjusted for lifestyle factors), high self-efficacy was associated with lower prevalence of CLS partners, STI diagnosis and PEP use, but not high partner numbers or group sex (Fig. 2). Similar, albeit weaker, associations were found when investigating difficulty negotiating condom use (Fig. 3).

Among men who reported CLS, men with depressive symptoms were more likely than those without to report having gotten carried away or been under the influence of alcohol or drugs (54.8\% v. 41.3\%; $P=0.005)$, their or their partner's difficulty in keeping an erection or ejaculating with a condom $(32.3 \%$ v. $22.6 \% ; P=0.020)$ and their partner not wanting to use a condom $(23.4 \%$ v. $16.2 \% ; P=0.050)$.

\section{Receiving medical treatment or therapy for depression}

Overall, the proportion of men who reported receiving treatment (medicine or therapy) for depression was 9.2\% (123 of 1340). Treatment for depression was reported by $28.9 \%(n=48)$ of the 166 men with depressive symptoms and $6.4 \%(n=75)$ of the 1174 men without. To more accurately present the prevalence of men with depression who were receiving treatment, men with either depressive symptoms (PHQ-9 $\geq 10$ ) or who reported receiving treatment for depression were considered to account for all men with any evidence of current depression. Among these 241 men, $51.0 \%(n=123)$ were receiving treatment for depression.

The prevalence of CLS partner measures, STI diagnosis and PEP use according to evidence of depression (symptoms and/or treatment) is presented in Table 3. Men with any evidence of current depression tended to be more likely to report measures of sexual risk than those without evidence of depression. Adjusted PRs of CLS with one or more partners, STI diagnosis and PEP use were higher for men who reported depressive symptoms (with or without treatment) than for those who reported treatment but no current symptoms. For the other measures of CLS, prevalence was elevated for all three depression groups, compared with no depression. 
Table 2 Prevalence of sexual behaviour measures among 1340 MSM who reported anal or vaginal sex in the past 3 months

MSM reporting anal or vaginal sex in the past 3 months $(N=1340)$

\begin{tabular}{|c|c|}
\hline & $n(\%) 95 \% \mathrm{Cl}$ \\
\hline CLS with one or more partners (past 3 months) & 853 (63.7) 61.0-66.2 \\
\hline CLS with two or more partners (past 3 months) & 430 (32.1) 29.6-34.6 \\
\hline CLS with unknown/HIV-positive status partner(s) ${ }^{a}$ (past 3 months) & 474 (35.4) 32.9-38.0 \\
\hline Receptive CLS with unknown status partner(s) ${ }^{b}$ (past 3 months) & $187(14.0)$ 12.2-15.9 \\
\hline Self-reported bacterial STI diagnosis (past year) & 423 (31.6) 29.1-34.1 \\
\hline PEP use (past year) & 207 (15.5) 13.6-17.5 \\
\hline Eleven or more new sexual partners (past year) & 483 (36.0) 33.5-38.7 \\
\hline Group sex (past 3 months) & 496 (37.0) 34.5-39.6 \\
\hline \multicolumn{2}{|c|}{$\begin{array}{l}\text { CLS, condomless sex; MSM, men who have sex with men; PEP, post-exposure prophylaxis; STI, sexually transmitted infection. } \\
\text { a. Men who reported no CLS partners of unknown HIV status, and only one HIV positive CLS partner who was a long-term partner and with whom they 'thought the risks of catching HIV } \\
\text { were low because their partner was taking ART' were not counted as positive for this measure. } \\
\text { b. Includes men who reported always being the receptive partner and those who reported being sometimes the receptive and sometimes insertive partner. Only men who reported that } \\
\text { they did not know the HIV status of any of their partners could be included, as for those who reported knowing some or all of their partners' HIV status, it was not possible to } \\
\text { distinguish whether this was the HIV-positive partner with whom the participant had insertive or receptive CLS. }\end{array}$} \\
\hline
\end{tabular}

\section{Discussion}

AURAH is the largest questionnaire study of sexual behaviour undertaken among individuals attending GUM clinics in the
UK and one of the first UK studies to assess the association of depressive symptoms with CLS. Depressive symptoms were associated with all measures of CLS, including bacterial STI diagnosis and PEP use, independently of sociodemographic and
N=1340 MSM reporting anal/vaginal sex (past 3 months)

Dependent variable:

CLS with $1+$ partners (past 3 months) $(n=853 ; 63.7 \%)$

CLS with 2+ partners (past 3 months) $(n=430 ; 32.1 \%)$

CLS with unknown/HIV+ partner(s) ${ }^{a}$ (past 3 months) $(n=474 ; 35.4 \%)$

Receptive CLS with unknown status partner(s) (past 3 months) $(n=187 ; 14.0 \%)$

Bacterial STI diagnosis (past year) $(n=423 ; 31.6 \%)$

PEP use (past year) $(n=207 ; 15.5 \%)$

$11+$ new sexual partners (past year) $(n=483 ; 36.0 \%)$

Group sex (past 3 months) $(n=496 ; 37.0 \%)$
Prevalence ratio associated

with PHQ-9 score $\geq 10$

(independent variable)

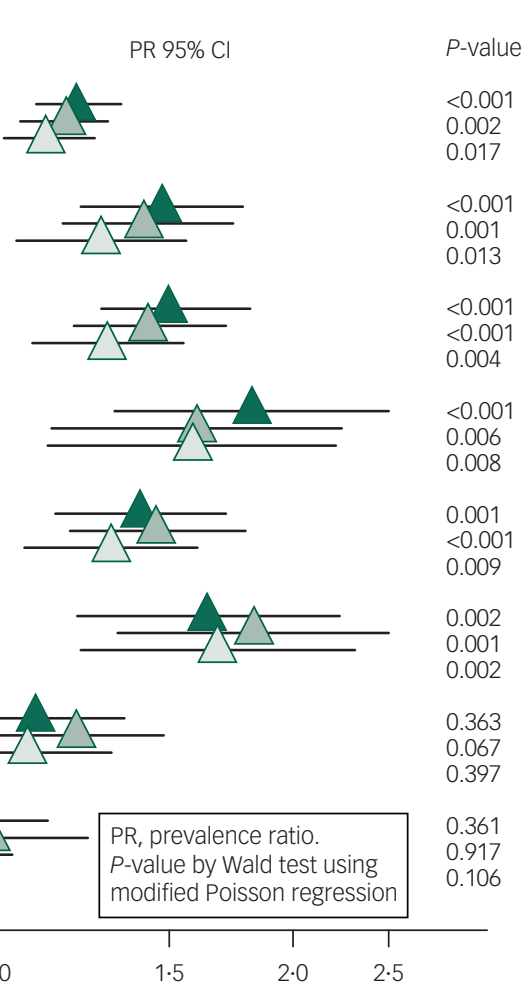

\footnotetext{
Unadjusted prevalence ratio $(N=1340)$

$\triangle$ Prevalence ratio adjusted for: age, $\mathrm{UK}$ born ${ }^{\mathrm{b}}$, sexual identity ${ }^{\mathrm{b}}$, university education, ongoing relationship status, and study region $(N=1316)$

$\triangle$ Prevalence ratio adjusted for above sociodemographic factors plus smoking, higher-risk drinking, and number of recreational drugs used $(N=1316)$
}

Fig. 1 Unadjusted and adjusted associations of current depressive symptoms on PHQ-9 $(\geq 10)$ with sexual behaviours among 1340 MSM who reported anal or vaginal sex in the past 3 months.

CLS, condomless sex; MSM, men who have sex with men; PEP, post-exposure prophylaxis; STI, sexually transmitted infection.

a. Excludes men who reported no CLS partners of unknown HIV status, and only one HIV positive CLS partner who was a long-term partner and with whom they 'thought the risks of catching HIV were low because their partner was taking ART'.

b. The model was fitted to include age in four categories $(<25 ; 25-29 ; 30-39 ; 40+)$, dichotomous UK born and self-reported sexual identity. 


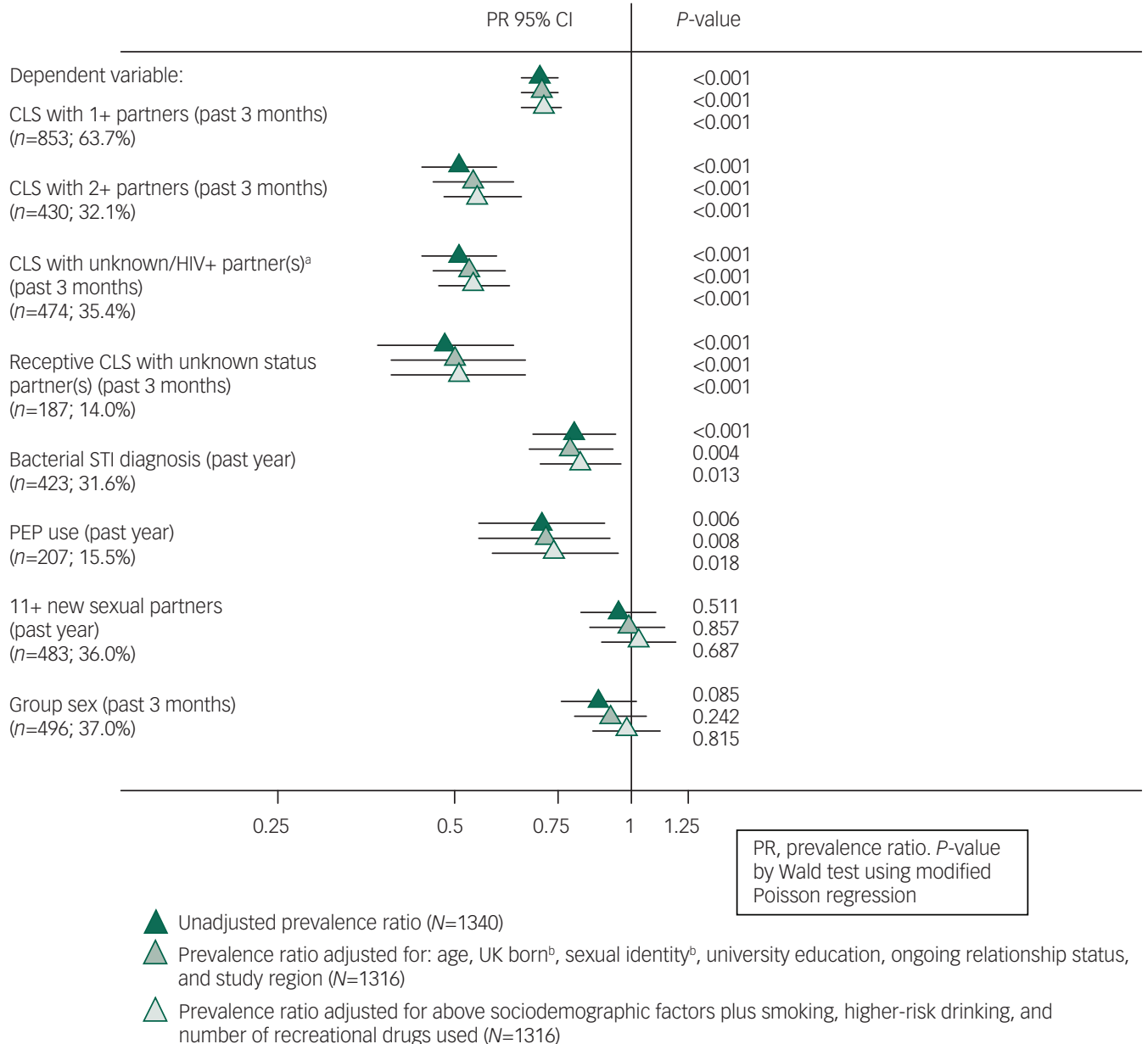

Fig. 2 Unadjusted and adjusted associations of high self-efficacy for sexual safety with sexual behaviours among 1340 MSM who reported anal or vaginal sex in the past 3 months.

CLS, condomless sex; MSM, men who have sex with men; PEP, post-exposure prophylaxis; STI, sexually transmitted infection.

a. Excludes men who reported no CLS partners of unknown HIV status, and only one HIV positive CLS partner who was a long-term partner and with whom they 'thought the risks of catching HIV were low because their partner was taking ART'.

b. The model was fitted to include age in four categories $(<25 ; 25-29 ; 30-39 ; 40+)$, dichotomous UK born and self-reported sexual identity.

lifestyle factors. However, depressive symptoms were not associated with having a higher number of new sexual partners or group sex.

\section{Burden of depressive symptoms among MSM and associated factors}

Minority stress theory and masculine socialisation stress theory stipulate that gay (and bisexual) men may experience high levels of psychological distress as a result of conflict produced between mainstream social norms and minority values. Social pressure to conform to traditional gender-normative behaviour may increase shaming and victimisation of men in sexual minority groups, and risk of internalised homophobia, adverse mental health outcomes, violence-prone partnerships and/or substance use for these men. ${ }^{33,34}$ A recent systematic review of mainly US or Canadian studies reported elevated levels of depression among sexual minorities compared with heterosexual individuals, ${ }^{35}$ but there is less evidence from the UK. In this study, the prevalence of depressive symptoms among MSM in 2013-2014 was 12\%. A general population-based survey in England in 2007-2008 found the prevalence of depressive symptoms to be $7 \%$ by the same definition (PHQ-9 $\geq 10$ ) and this figure included women, among whom rates of depression are often higher. This suggests prevalence may be somewhat higher among MSM compared with other men, but other sociodemographic factors may confound this comparison. ${ }^{36}$ There are a number of other probabilitybased sample and general population-based survey studies that have collected information on depression among individuals in the UK; however, none have incorporated the PHQ-9. ${ }^{37-42}$ In the most recent (2010-2012) British National Survey of Sexual Attitudes and Lifestyles (NATSAL), a shortened version of the PHQ-9 was used (PHQ-2, which includes only the first two questions): $8.9 \%$ (95\% CI $5.5-14.3, n / N=17 / 190)$ of all MSM, $6.4 \%$ (95\% CI 3.5-11.7, $n / N=7 / 107)$ of gay-identified MSM and $8.9 \%$ (95\% CI 8.1-9.8, $n / N=451 / 5069)$ of men who reported sex exclusively with women screened positive for depression. ${ }^{43}$ By the same definition (PHQ-2 $\geq 3$ ), the prevalence of depression in MSM included in this AURAH analysis was 9.3\%; among all MSM in AURAH the comparable prevalence was 9.5\%.

Studies from the UK, USA and Australia have found that among MSM, markers of lower socioeconomic status and lower levels of a supportive network are consistently strongly associated with 


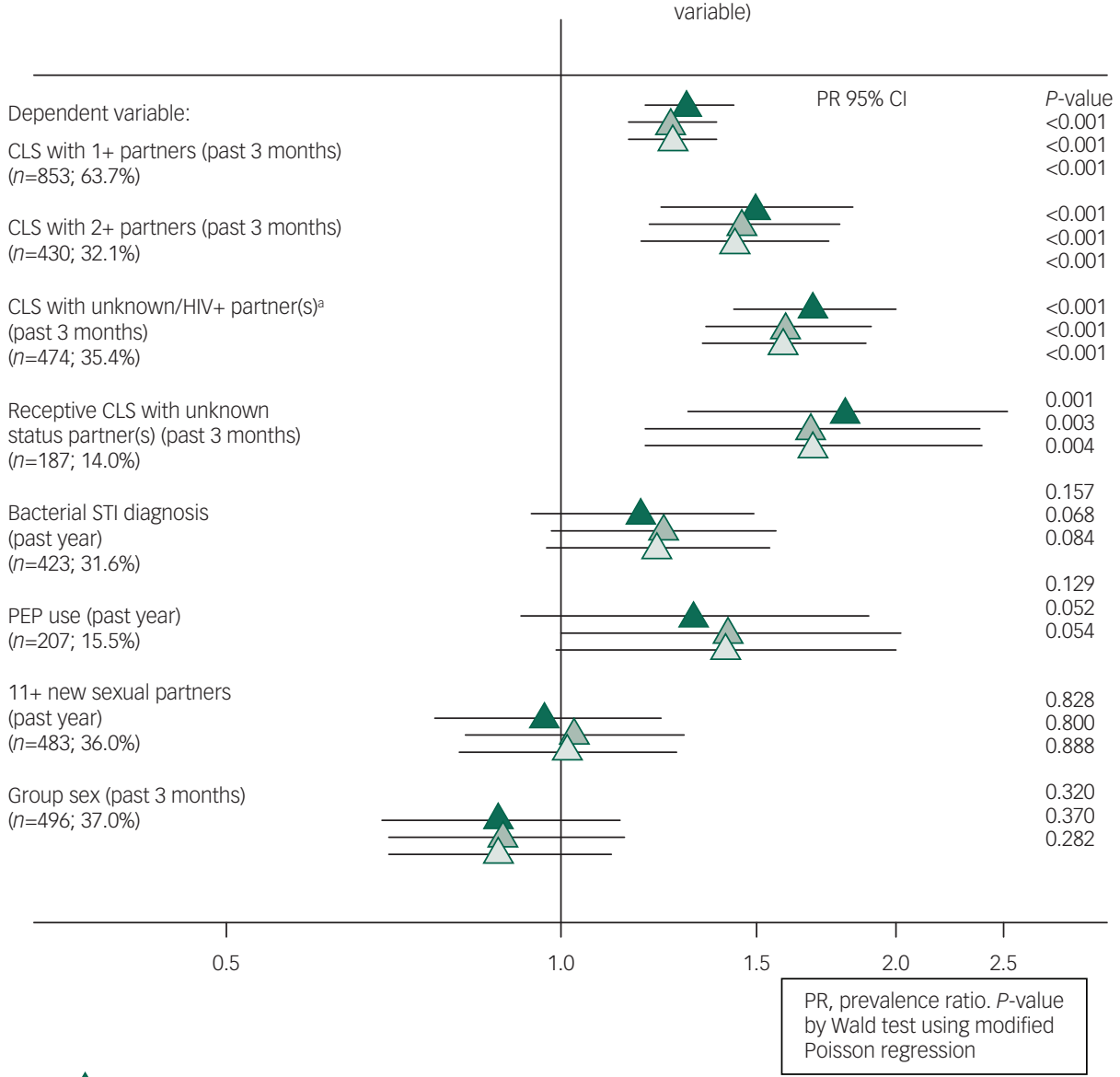

$\Delta$ Unadjusted prevalence ratio $(N=1340)$

Poisson regression

$\triangle$ Prevalence ratio adjusted for: age, UK born' ${ }^{b}$, sexual identity ${ }^{b}$, university education, ongoing relationship status, and study region $(N=1316)$

$\triangle$ Prevalence ratio adjusted for above sociodemographic factors plus smoking, higher-risk drinking, and number of recreational drugs used $(N=1316)$

Fig. 3 Unadjusted and adjusted associations of finding it difficult to negotiate condom use with sexual behaviours among 1340 MSM who reported anal or vaginal sex in the past 3 months.

CLS, condomless sex; MSM, men who have sex with men; PEP, post-exposure prophylaxis; STI, sexually transmitted infection.

a. Excludes men who reported no CLS partners of unknown HIV status, and only one HIV positive CLS partner who was a long-term partner and with whom they 'thought the risks of catching HIV were low because their partner was taking ART'.

b. The model was fitted to include age in four categories $(<25 ; 25-29 ; 30-39 ; 40+)$, dichotomous UK born and self-reported sexual identity.

symptoms of depression. ${ }^{44-46}$ Similarly, in this study these factors were among the strongest correlates of depressive symptoms, suggesting a critical role of socioeconomic hardship in poor mental health among UK MSM, as has been found in the general population. ${ }^{42,47,48}$

Younger age and recreational drug use were associated with depressive symptoms among MSM in AURAH, as has been found in other studies. ${ }^{44,46}$ The prevalence of depressive symptoms was twice as high in men reporting a bisexual (or other plurisexual) identity compared with a gay sexual identity $(22.1 \%$ v. $11.4 \%$, respectively), a finding that has also been reported in other studies. ${ }^{46,49}$ This may reflect higher levels of social support available in a more established and potentially more cohesive community, such as the gay community. Bisexual men may be even more vulnerable to isolation as a sexual minority. Low levels of disclosure of sexual orientation to work colleagues, smoking and higher risk alcohol use were also associated with depressive symptoms.

\section{Depressive symptoms and sexual behaviour}

To date, two European studies have investigated the relationship between symptoms of depression and sexual behaviour among MSM.
In a UK GUM clinic sample of 123 MSM in 1999, a higher prevalence of depressive symptoms (Hospital Anxiety and Depression Score (HADS) $\geq 11$ ) was observed among men who reported CLS with an HIV-positive or unknown status partner $v$. other men $\left(\chi^{2}\right.$ test $13.6 \%$ v. $2.7 \%$, based on eight cases of depression only). ${ }^{3}$ In a Belgian volunteer and online sample of 591 HIV-negative MSM who reported anal sex with a casual partner (2008), depressive symptoms (CES-D >21) were associated with CLS (odds ratio (OR) 1.66, 95\% CI 1.16-2.37; $P=0.006$ ) in unadjusted analysis. ${ }^{5}$ In US studies, the association between depressive symptoms and sexual risk among MSM has been more consistently demonstrated among samples where the vast majority of men reported recent sex and recreational drug use (Project MIX and Frontiers in Prevention ${ }^{7,9}$ ), compared with samples which included a higher proportion of men who did not report recent sex (Urban Men's Health Study and Sex and Love Study ${ }^{15,21}$ ). It has been suggested that in the former samples, men may have been more likely to have an externalised response to depressive symptoms (such as cognitive escape) rather than an internalised response such as withdrawal. ${ }^{7}$ This may in part explain why a 


\begin{tabular}{|c|c|c|c|c|c|c|}
\hline \multicolumn{3}{|c|}{$N=1340$ MSM reporting sex in the past 3 months } & \multirow[b]{2}{*}{$N(\%)$} & \multirow[b]{2}{*}{$\begin{array}{l}\% \\
p^{\mathrm{a}}\end{array}$} & \multirow[b]{2}{*}{$\begin{array}{l}\text { Unadjusted PR (95\% Cl) } \\
\qquad P^{\mathrm{b}}\end{array}$} & \multirow[b]{2}{*}{$\begin{array}{c}\text { Adjusted PR }(95 \% \mathrm{Cl}) \\
\qquad P^{\mathrm{b}}\end{array}$} \\
\hline & $\begin{array}{l}\text { Depressive symptoms } \\
\quad(\mathrm{PHQ}-9 \geq 10)\end{array}$ & $\begin{array}{l}\text { Receiving treatment } \\
\text { for depression }\end{array}$ & & & & \\
\hline \multirow{5}{*}{ CLS with one or more partners } & Yes & Yes & $48(3.6)$ & $81.3 \%$ & $1.32(1.14,1.52)$ & $1.26(1.08,1.46)$ \\
\hline & Yes & No & $118(8.8)$ & $72.0 \%$ & $1.17(1.03,1.32)$ & $1.30(1.02,1.30)$ \\
\hline & No & Yes & $75(5.6)$ & $68.0 \%$ & $1.10(0.94,1.30)$ & $1.06(0.90,1.24)$ \\
\hline & No & No & 1099 (82.0) & $61.7 \%$ & 1 & 1 \\
\hline & & & & 0.006 & $<0.001$ & 0.006 \\
\hline \multirow[t]{5}{*}{ CLS with two or more partners } & Yes & Yes & $48(3.6)$ & $54.2 \%$ & $1.83(1.39,2.41)$ & $1.65(1.23,2.21)$ \\
\hline & Yes & No & $118(8.8)$ & $40.7 \%$ & $1.37(1.08,1.74)$ & $1.37(1.08,1.75)$ \\
\hline & No & Yes & $75(5.6)$ & $40.0 \%$ & $1.35(1.01,1.81)$ & $1.35(1.01,1.80)$ \\
\hline & No & No & 1099 (82.0) & $29.7 \%$ & 1 & 1 \\
\hline & & & & $<0.001$ & $<0.001$ & 0.001 \\
\hline \multirow{5}{*}{$\begin{array}{l}\text { CLS with unknown/HIV-positive } \\
\text { status partner(s) }{ }^{c}\end{array}$} & Yes & Yes & $48(3.6 \%)$ & $54.2 \%$ & $1.69(1.29,2.22)$ & $1.50(1.11,2.02)$ \\
\hline & Yes & No & $118(8.8 \%)$ & $49.2 \%$ & $1.53(1.25,1.88)$ & $1.48(1.20,1.82)$ \\
\hline & No & Yes & 75 (5.6\%) & $50.7 \%$ & $1.58(1.25,2.01)$ & $1.57(1.23,2.00)$ \\
\hline & No & No & 1099 (82.0\%) & $32.0 \%$ & 1 & 1 \\
\hline & & & & $<0.001$ & $<0.001$ & $<0.001$ \\
\hline \multirow{5}{*}{$\begin{array}{l}\text { Receptive CLS with an unknown } \\
\text { status partner }\end{array}$} & Yes & Yes & $48(3.6)$ & $25.0 \%$ & $2.10(1.25,3.51)$ & $1.68(0.94,2.99)$ \\
\hline & Yes & No & $118(8.8)$ & $22.0 \%$ & $1.85(1.27,2.69)$ & $1.71(1.16,2.52)$ \\
\hline & No & Yes & $75(5.6)$ & $24.0 \%$ & $2.01(1.30,3.11)$ & $2.08(1.34,3.21)$ \\
\hline & No & No & 1099 (82.0) & $11.9 \%$ & 1 & 1 \\
\hline & & & & $<0.001$ & $<0.001$ & 0.001 \\
\hline \multirow{5}{*}{$\begin{array}{l}\text { Self-reported bacterial STI } \\
\text { diagnosis (past year) }\end{array}$} & Yes & Yes & $48(3.6)$ & $35.4 \%$ & $1.19(0.80,1.76)$ & $1.34(0.91,1.96)$ \\
\hline & Yes & No & $118(8.8)$ & $44.9 \%$ & $1.50(1.21,1.87)$ & $1.52(1.21,1.92)$ \\
\hline & No & Yes & $75(5.6)$ & $33.3 \%$ & $1.12(0.80,1.56)$ & $1.12(0.81,1.55)$ \\
\hline & No & No & $1099(82.0)$ & $29.9 \%$ & 1 & 1 \\
\hline & & & & 0.009 & 0.004 & 0.003 \\
\hline \multirow[t]{5}{*}{ PEP use (past year) } & Yes & Yes & $48(3.6)$ & $25.0 \%$ & $1.77(1.06,2.96)$ & $2.05(1.21,3.47)$ \\
\hline & Yes & No & $118(8.8)$ & $22.9 \%$ & $1.62(1.13,2.33)$ & $1.78(1.22,2.58)$ \\
\hline & No & Yes & $75(5.6)$ & $17.3 \%$ & $1.23(0.73,2.06)$ & $1.28(0.77,2.12)$ \\
\hline & No & No & $1099(82.0)$ & $14.1 \%$ & 1 & 1 \\
\hline & & & & 0.018 & 0.014 & 0.002 \\
\hline \multicolumn{7}{|c|}{$\begin{array}{l}\text { Note: Adjusted models - age (in four categories: }<25,25-29,30-39 \text { or } \geq 40 \text { years), UK born (yes or no/missing), sexual identity (gay or bisexual/straight), university education (yes or no/ } \\
\text { missing), ongoing relationship status (yes or no//missing) and study region (London, South or other). } \\
\text { CLS, condomless sex; MSM, men who have sex with men; PEP, post-exposure prophylaxis; PHQ, Patient Health Questionnaire; PR, prevalence ratio; STI, sexually transmitted infection. } \\
\text { a. Pearson's } \chi^{2} \text { test. } \\
\text { b. Wald test from modified Poisson regression. } \\
\text { c. Excludes men who reported no CLS partners of unknown HIV status, and only one HIV positive CLS partner who was a long-term partner and with whom they 'thought the risks of } \\
\text { catching HIV were low because their partner was taking ART'. }\end{array}$} \\
\hline
\end{tabular}

strong relationship was observed in this study among a sexually active GUM clinic sample.

\section{Depression, alcohol and drug use and sexual behaviour}

Our findings suggest that the link between depression and CLS may in part be confounded by higher levels of drug or alcohol use among those with depression, but drug and alcohol use are unlikely to solely explain this relationship. It has been further stipulated in syndemic theory that it is the synergistic interactions of two or more co-occurring psychosocial health problems, such as depression, drug use, childhood sexual abuse and intimate partner violence, that compound the risk of HIV infection among MSM. ${ }^{21}$ Measurement of these factors together with markers of certain personality traits (sensation seeking and sexual compulsivity) would be useful in further studies.

\section{Self-efficacy for sexual safety}

Our findings suggest that low self-efficacy for sexual safety and difficulty negotiating condom use may be potential mechanisms through which depression leads to CLS, which may explain why a relationship was observed with CLS measures but not higher partner numbers. The fact that men who reported depressive symptoms were more likely to report PEP use suggests that although depression may impair discussion or negotiation of safe sex practices, there is still an overarching desire to be safe and protect oneself after this interaction is over.

\section{Symptoms and treatment for depression in relation to sexual risk}

In this study, there was limited evidence to imply that sucessful treatment of depression (reporting treatment, but negative for depressive symptoms according to PHQ-9) may reduce sexual risk. However, the extent to which this question can be addressed given the cross-sectional design of the study is limited, and inference may be complicated by a number of factors including severity of symptoms before treatment, type, duration and frequency of treatment, timing of treatment in relation to symptoms, the presence of other psychological conditions and sensitivity of the PHQ-9 in capturing all cases of depressive symptoms. There does appear to be some evidence of potential undertreatment of possible clinically significant depressive conditions.

\section{Strengths and limitations}

Strengths of this study include the large sample size and the fact that only men who reported sex in the past 3 months were included in analysis. This is important, as in some men depression may result in lowered libido and lack of sexual activity. In terms of limitations, the prevalence of depression and associations with sexual behaviour may have differed among the $40 \%$ of individuals approached who did not complete the questionnaire. Furthermore, the cross-sectional methodology used in this study prohibits us from making inferences about causality. In particular, the PHQ-9 inquires about symptoms during the previous 2 weeks, 
whereas the recall periods for sexual behaviour were longer (past 3 months or past year). Because depression questionnaires aim to provide a diagnostic measure of a chronic mental health condition, it is not unreasonable to assume that in many participants depression may have preceded the recent sexual behaviour reported. The strong adjusted associations found between depressive symptoms and CLS, and the evidence for low self-efficacy as a mechanism for these relationships, provide some support in favour of a causal association. Furthermore, there appeared to be some evidence of a gradient in CLS prevalence with more severe depressive symptoms. On the other hand, the elevated prevalence of some CLS measures among men who reported treatment for depression but were not PHQ-9 positive (i.e. apparently successful treatment) could be regarded as evidence that causality, at least to some extent, may operate in the other direction. Finally, our study is important in the context of GUM services but it does not necessarily allow us to generalise to all MSM in the UK.

\section{Implications}

Our findings suggest the need for an integrated sexual health approach. Screening for depressive symptoms using brief instruments in sexual health services may be useful in identifying individuals who could be offered referral for potential interventions. Socioeconomic advantage and supportive networks may be critical factors for mental health. Care should be taken to include men with plurisexual identities in efforts to promote supportive networks and manage symptoms of depression. In parallel, integration of (or good linkage to) substance use services could also play a role in management of depression and reduction of sexual risk.

Ada R. Miltz, MSC, HIV Epidemiology and Biostatistics Group, Research Department of Infection and Population Health, University College London, London, UK; Alison J. Rodger, MD, HIV Epidemiology and Biostatistics Group, Research Department of Infection and Population Health, University College London, London, UK; Janey Sewell, BNurs, HIV Epidemiology and Biostatistics Group, Research Department of Infection and Population Health, University College London, London, UK; Andrew Speakman, PhD, HIV Epidemiology and Biostatistics Group, Research Department of Infection and Population Health, University College London, London, UK; Andrew N. Phillips, PhD, HIV Epidemiology and Biostatistics Group, Research Department of Infection and Population Health, University College London, London, UK; Lorraine Sherr, PhD, HIV Epidemiology and Biostatistics Group, Research Department of Infection and Population Health, University College London, London, UK; Richard J. Gilson, MBBS, Centre for Sexual Health and HIV Research, Research Department of Infection and Population Health, Mortimer Market Centre, University College London, London, UK; David Asboe, MBBS, John Hunter Clinic, London, UK; Nneka C. Nwokolo, MBBS, 56 Dean Street, London, UK; Amanda Clarke, BM, Royal Sussex County Hospital, Brighton, UK; Mark M. Gompels, MBBS, Southmead Hospital, Bristol, UK; Sris Allan MBBS, City of Coventry Healthcare Centre, Coventry, UK Simon Collins, advocate, HIV i-Base, London, UK; Fiona C. Lampe, PhD, HIV Epidemiology and Biostatistics Group, Research Department of Infection and Population Health, University College London, London, UK, for the AURAH Study Group.

Correspondence: Ada R. Miltz, HIV Epidemiology and Biostatistics Group, Research Department of Infection and Population Health, University College London, London, UK. Email: Ada.Miltz.11@ucl.ac.uk

First received 14 Jul 2016, final revision 13 Jan 2017, accepted 6 Mar 2017

\section{Acknowledgements}

We thank all study participants for their time and effort. We gratefully acknowledge the contributions of all the AURAH clinic teams who helped with data collection, distribution of questionnaires and administrative tasks. The AURAH study group: Ada R. Miltz, Alison J. Rodger, Andrew Speakman, Fiona C Lampe, Andrew N. Phillips, Janey Sewell, Lorraine Sherr, Richard J. Gilson, David Asboe, Nneka C. Nwokolo, Amanda Clarke, Mark M. Gompels, Sris Allan, Simon Collins, Christopher Scott, Sara Day, Martin Fisher, Jane Anderson, Rebecca O'Connell, Monica Lascar, Vanessa Apea, Maneh Farazmand, Susan Mann, Jyoti Dhar, Daniel R. Ivens, Tariq Sadiq, Stephen Taylor, Michael Brady, Alan Tang, Rageshri Dhairyawan, Graham J. Hart, Anne M. Johnson, Alec Miners, and Jonathan Elford. AURAH clinic teams: Rageshri Dhairyawan, Sharmin Obeyesekera (Barking), Vanessa Apea, John Saunders, James Hand, Nyasha Makoka (Barts and the London), Stephen Taylor, Gerry Gilleran, Cathy Stretton (Birmingham), Martin Fisher, Amanda Clarke, Nicky Perry, Elaney Youssef, Celia Richardson, Louise Kerr, Mark Roche, David Stacey, Sarah Kirk (Brighton), Mark Gompels, Louise Jennings, Caroline Holder, Katie Anne Baker (Bristol), Maneh Farazmand Matthew Robinson, Emma Stree (Calderdale \& Huddersfield), Sris Allan, Abayomi Shomoye (Coventry), Nneka Nwokolo,
Ali Ogilvy (Dean Street), Jane Anderson, Sfiso Mguni, Rebecca Clark, Cynthia Sajani, Veronica Espa (Homerton), David Asboe, Sara Day, Ali Ogilvy, Sarah Ladd (John Hunter), Susan Mann, Michael Brady, Jonathan Syred, Lisa Hamza, Lucy Campbell, Emily Wandolo, Janagan Alagarajah (Kings), Linda Mashonganyika, Jyoti Dhar, Sally Batham (Leicester), Richard Gilson, Rita Trombin, Ana Milinkovic, Clare Oakland (Mortimer Market), Rebecca O'Connell, Nyasha Makoka (Newham), Alan Tang, Ruth Wilson, Elizabeth Green, Sheila O'Connor, Sarah Kempster, Katie Keating-Fedders (Reading), Daniel Ivens, Nicola Tyrrell, Jemima Rogers, Silvia Belmondo, Manjit Sohal (Royal Free), Tariq Sadiq, Wendy Majewska, Anne Patterson, Olanike Okolo, David Cox, Mariam Tarik, Charlotte Jackson, Jeanette Honigsbaum, Clare Boggon, Simone Ghosh, Bernard Kelly, Renee Aroney (St George's), Christopher Scott, Ali Ogilvy (West London Clinic for Sexual Health), and Monica Lascar, Nyasha Makoka, Elias Phiri, Zandile Maseko (Whipps Cross). AURAH (core) Study Group: Alison Rodger, Fiona Lampe, Andrew Phillips and Andrew Speakman. AURAH data managers: Andrew Speakman and Ada Miltz. AURAH study nurse coordinator: Janey Sewell. AURAH advisory board: Lorraine Sherr, Graham Hart, Simon Collins, Anne Johnson, Alec Miners and Jonathan Elford. CAPRA advisory board: Sir Nick Partridge, Kay Orton, Anthony Nardone, and Ann Sullivan.

\section{Funding}

The AURAH study was funded by the National Institute for Health Research (NIHR) under its Programme Grants for Applied Research funding scheme (RP-PG-0608-10142). The views expressed in this report are those of the authors and not necessarily those of the NHS, the NIHR or the Department of Health. The AURAH Study Group acknowledges the support of the NIHR, through the Comprehensive Clinical Research Network. The sponsor of the study had no role in design and conduct of the study; collection, management, analysis and interpretation of the data; preparation, review or approval of the manuscript; and decision to submit the manuscript for publication.

\section{References}

1 Chau C, Kirwan P, Brown A, Gill N, Delpech V. HIV Diagnoses, Late Diagnoses and Numbers Accessing Treatment and Care. Public Health England, 2016.

2 Crepaz N, Marks G. Are negative affective states associated with HIV sexual risk behaviors? A meta-analytic review. Health Psychol 2001; 20: 291-9.

3 Beck A, MCNally I, Petrak J. Psychosocial predictors of HIV/STI risk behaviours in a sample of homosexual men. Sex Transm Infect 2003; 79: 142-6.

4 Paul JP, Catania J, Pollack L, Stall R. Understanding childhood sexual abuse as a predictor of sexual risk-taking among men who have sex with men: the urban men's health study. Child Abuse Negl 2001; 25: 557-84.

5 Vanden Berghe W, Nostlinger C, Laga M. Syndemic and other risk factors for unprotected anal intercourse among an online sample of Belgian HIV negative men who have sex with men. AIDS Behav 2014; 18: 50-8.

6 De Santis JP, Colin JM, Provencio Vasquez E, McCain GC. The relationship of depressive symptoms, self-esteem, and sexual behaviors in a predominantly Hispanic sample of men who have sex with men. Am J Men's Health 2008; 2: 314-21.

7 Alvy LM, McKirnan DJ, Mansergh G, Koblin B, Colfax GN, Flores SA, et al. Depression is associated with sexual risk among men who have sex with men, but is mediated by cognitive escape and self-efficacy. AIDS Behav 2011; 15: $1171-9$

8 Fendrich M, Avci O, Johnson TP, Mackesy-Amiti ME. Depression, substance use and HIV risk in a probability sample of men who have sex with men. Addict Behav 2013; 38: 1715-8.

9 Houston E, Sandfort T, Dolezal C, Carballo-Dieguez A. Depressive symptoms among MSM who engage in bareback sex: does mood matter? AIDS Behav 2012; 16: 2209-15.

10 Koblin BA, Husnik MJ, Colfax G, Huang $Y$, Madison M, Mayer $K$, et al. Risk factors for HIV infection among men who have sex with men. AIDS 2006; 20 : 731-9.

11 Tucker A, Liht J, de Swardt G, Jobson G, Rebe K, McIntyre J, et al. An exploration into the role of depression and self-efficacy on township men who have sex with men's ability to engage in safer sexual practices. AIDS Care 2013; 25: 1227-35.

12 Shiu CS, Chen YC, Tseng PC, Chung AC, Wu MT, Hsu ST, et al. Curvilinear relationship between depression and unprotected sexual behaviors among men who have sex with men. J Sex Med 2014; 11: 2466-73.

13 Mimiaga MJ, Noonan E, Donnell D, Safren SA, Koenen KC, Gortmaker S, et al. Childhood sexual abuse is highly associated with HIV risk-taking behavior and infection among MSM in the EXPLORE Study. J Acquir Immune Defic Syndr 2009; 51: $340-8$.

14 Reisner SL, Mimiaga MJ, Skeer M, Mayer KH. Beyond anal sex: sexual practices associated with HIV risk reduction among men who have sex with men in Boston, Massachusetts. AIDS Patient Care STDS 2009; 23: 545-50.

15 Stall R, Mills TC, Williamson J, Hart T, Greenwood G, Paul J, et al. Association of co-occurring psychosocial health problems and increased vulnerability to HIV/ AIDS among urban men who have sex with men. Am J Public Health 2003; 93 939-42. 
16 Plankey MW, Ostrow DG, Stall R, Cox C, Li X, Peck JA, et al. The relationship between methamphetamine and popper use and risk of HIV seroconversion in the multicenter AIDS cohort study. J Acquir Immune Defic Syndr 2007; 45. 85-92.

17 Perdue TE, Hagan $\mathrm{H}$, Thiede $\mathrm{H}$, Valleroy L. Depression and HIV risk behaviou among Seattle-area injection drug users and young men who have sex with men. AIDS Educ Prev 2003; 15: 81-92.

18 Dudley MG, Rostosky SS, Korfhage BA, Zimmerman RS. Correlates of high-risk sexual behavior among young men who have sex with men. AIDS Educ Prev 2004; 16: 328-40.

19 Klein $\mathrm{H}$. Depression and HIV risk taking among men who have sex with other men (MSM) and who use the Internet to find partners for unprotected sex. I Gay Lesbian Ment Health 2014; 18: 164-89.

20 Nelson KM, Simoni JM, Pearson CR, Walters KL. 'I've had unsafe sex so many times why bother being safe now?': The role of cognitions in sexual risk among American Indian/Alaska Native men who have sex with men. Ann Behav Med 2011; 42: 370-80.

21 Parsons JT, Grov C, Golub SA. Sexual compulsivity, co-occurring psychosocial health problems, and HIV risk among gay and bisexual men: further evidence of a syndemic. Am J Public Health 2012; 102: 156-62.

22 McKirnan DJ, Ostrow DG, Hope B. Sex, drugs and escape: a psychological mode of HIV-risk sexual behaviours. AIDS Care 1996; 8: 655-69.

23 Krohne HW. Attention and avoidance: strategies in coping with aversiveness. In Attention and Avoidance: Two Central Strategies in Coping with Aversiveness (ed HW Krohne): 3-15. Hogrefe and Huber, 1993.

24 Bandura A. Toward a unifying theory of behavioral change. Psychol Rev 1977; 84 $191-215$.

25 Sewell J, Speakman A, Phillips AN, Lampe FC, Miltz A, Gilson R, et al. A cross-sectional study on attitudes to and understanding of risk of acquisition of HIV: design, methods and participant characteristics. JMIR Res Protoc 2016; 5: e58.

26 Galupo MP, Lomash E, Mitchell RC. 'All of My Lovers Fit into This Scale': sexual minority individuals' responses to two novel measures of sexual orientation. J Homosex 2017; 64: 145-65.

27 Kroenke K, Spitzer RL, Williams JB. The PHQ-9: validity of a brief depression severity measure. J Gen Intern Med 2001; 16: 606-13.

28 Zou G. A modified poisson regression approach to prospective studies with binary data. Am J Epidemiol 2004; 159: 702-6.

29 StataCorp. STATA Statistical Software. 13th ed. StataCorp, 2009

30 STROBE. Strobe Statement - Checklist of Items that Should be Included in Reports of Cross-Sectional Studies. University of Bern, 2009 (http://www.strobestatement.org/fileadmin/Strobe/uploads/checklists/STROBE_checklist_v4_crosssectional.pdf).

31 Broadhead WE, Gehlbach SH, de Gruy FV, Kaplan BH. The duke-UNC functional social support questionnaire. Measurement of social support in family medicine patients. Med Care 1988; 26: 709-23.

32 Babor TF, Higgins-Biddle JC, Saunders JB, Monteiro MG. The Alcohol Use Disorders Identification Test. Guidelines for Use in Primary Care. Department of Mental Health and Substance Dependence, World Health Organization, 2001

33 Stall $R$, Friedman M, Catania JA. Interacting epidemics and gay men's health: a theory of syndemic production among urban gay men. In Unequal Opportunity: Health Disparaties Affecting Gay and Bisexual Men in the United States (eds RJ Wolitski, R Stall, RO Valdiserri): 222-236. Oxford University Press, 2008

34 Meyer IH. Prejudice, social stress, and mental health in lesbian, gay, and bisexual populations: conceptual issues and research evidence. Psychol Bull 2003; 129. 674-97.

35 Ploderl M, Tremblay P. Mental health of sexual minorities. A systematic review. Int Rev Psychiatry 2015; 27: 367-85.

36 Paranjothy S, Gallacher J, Amlot R, Rubin GJ, Page $L$, Baxter $T$, et al. Psychosocial impact of the summer 2007 floods in England. BMC Public Health 2011; 11: 145

37 Stewart R, Prince M, Harwood R, Whitley R, Mann A. Quality of accommodation and risk of depression in later life: an analysis of prospective data from the Gospel Oak Project. Int J Geriatr Psychiatry 2002; 17: 1091-8.

38 Campbell R, Mills N, Sanford E, Graham A, Low N, Peters TJ. Does population screening for Chlamydia trachomatis raise anxiety among those tested? Findings from a population based chlamydia screening study. BMC Public Health 2006: 6: 106.

39 Sham PC, Sterne A, Purcell S, Cherny S, Webster M, Rijsdijk F, et al. GENESiS: creating a composite index of the vulnerability to anxiety and depression in a community-based sample of siblings. Twin Res 2000; 3: 316-22.

40 Ohayon MM, Priest RG, Guilleminault C, Caulet M. The prevalence of depressive disorders in the United Kingdom. Biol Psychiatry 1999; 45: 300-7.

41 Bebbington $P E$, Marsden $L$, Brewin $C R$. The need for psychiatric treatment in the general population: the Camberwell Needs for Care Survey. Psychol Med 1997: 27: 821-34.

42 Bebbington $\mathrm{P}$, Brugha T, Coid J, Crawford M, Deverill C, D'Souza J, et al. Adult Psychiatric Morbidity in England, 2007. Results of a Household Survey. The National Centre for Social Research and the Department of Health Sciences, University of Leicester. The Health \& Social Care Information Centre, Social Care Statistics, 2009.

43 Mercer $\mathrm{CH}$, Prah $\mathrm{P}$, Field $\mathrm{N}$, Tanton $\mathrm{C}$, Macdowall $\mathrm{W}$, Clifton $\mathrm{S}$, et al. The health and well-being of men who have sex with men (MSM) in Britaln: evidence from the third National Survey of Sexual Attitudes and Lifestyles (NATSAL-3). BMC Public Health 2016; 16: 525

44 Mao L, Kidd MR, Rogers G, Andrews G, Newman CE, Booth A, et al. Social factors associated with Major Depressive Disorder in homosexually active, gay men attending general practices in urban Australia. Aust N Z J Public Health 2009; 33: $83-6$

45 Ingram KM, Jones DA, Fass RJ, Neidig JL, Song YS. Social support and unsupportive social interactions: their association with depression among people living with HIV. AIDS Care 1999; 11: 313-29.

46 Hickson F, Davey C, Reid D, Weatherburn P, Bourne A. Mental health inequalities among gay and bisexual men in England, Scotland and Wales: a large communitybased cross-sectional survey. I Public Health 2016; doi: 10.1093/pubmed/fdw021 (Epub ahead of print)

47 Fone D, Dunstan F, Williams G, Lloyd K, Palmer S. Places, people and mental health: a multilevel analysis of economic inactivity. SOC Sci Med 2007; 64: 633-45

48 Stafford M, Marmot M. Neighbourhood deprivation and health: does it affect us all equally? Int J Epidemiol 2003; 32: 357-66.

49 Marshal MP, Dietz $\amalg$, Friedman MS, Stall R, Smith HA, McGinley J, et al. Suicidality and depression disparities between sexual minority and heterosexual youth: a meta-analytic review. J Adolesc Health 2011; 49: 115-23. 